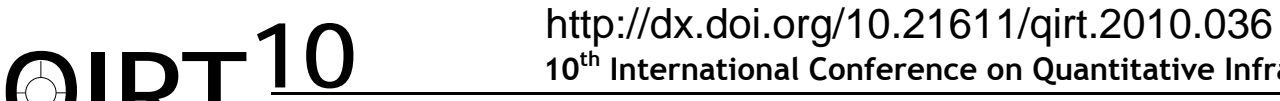 \\ $10^{\text {th }}$ International Conference on Quantitative InfraRed Thermography \\ July 27-30, 2010, Québec (Canada)
}

\section{Application of the morphological operations for defect area recognition using active thermography}

by S. Dudzik*

\author{
*Faculty of Electrical Engineering, Czestochowa University of Technology, Poland, sebdud@el.pcz.czest.pl
}

\begin{abstract}
In paper some examples of the application of the morphological operations in defect detection, using an active infrared thermography are described. The fundamentals of the mathematical morphology and the two methods of the global thresholding are presented. These methods were used to the binarization of the analyzed thermal images. Further, the results of the background estimation, using an opening operation were presented. The estimation was performed for the thermal images of the defected material sample. The sample had a few defects with different depths. The experimental research were carried out using the thermal image sequence, recorded with the stepped heating method.
\end{abstract}

\section{Introduction}

An active infrared thermography is a very interesting method for defect detection, especially when defects lie near the surface of investigated specimen [1-4]. In the active scheme, the heat excitation is deposited on the specimen surface and the temporal response is recorded. In order to obtain a temperature distribution, the infrared camera is used most frequently [5]. However it is only the first stage of the investigations. Then, it is necessary to process the recorded time sequence of thermal images [6]. For this purpose, the sophisticated image processing procedures are applied to the recorded thermograms. There are known the methods operating in the time and frequency domain, but in each case, the specialized software must be employed in order to retrieval of the information about flaws existing in the examined sample. In this work the morphological operations are applied for recognition of defected areas. The theoretical basis of mathematical morphology is presented. Further it is described the application of the morphological opening operation for the background estimation and two thresholding methods for thermal image binarization as well. In paper the results of experiments conducted in MATLAB environment are presented. Investigations were carried out using the thermal image sequence recorded with the stepped heating method, for sample with a low thermal diffusivity.

\section{Mathematical morphology and binarization methods in thermal image processing}

\subsection{Elementary morphological operations}

Mathematical morphology is a very important tool in the computer-aided image processing [6]. It enables complex operations attached with shape and mutual arrangement analysis of objects existing in the investigated scene. The fundamental idea of morphology in image processing is that an image is considered as a set (binary images) or simply function (intensity images). A very important conception is a structuring element. This element is applied to an input image and next, an output image is generated. The basic morphological operations used in this work are erosion, dilation and morphological opening. These operations are applied to the thermal images come from the thermal images sequence. The main purpose of use this operations (especially opening) is an estimation of the background of investigated thermal images. The background estimation and additional image processing routines were conducted in order to detect flaws existed in examined sample.

The elementary morphological operations for the intensity images $J(x, y)$ are presented below. Dilation of the $J(x, y)$ image can be expressed as [7]:

$$
D(J(x, y), B(r, s))=\max _{r, s \in B(x, y)}\{J(x-r, y-s)+B(r, s)\}
$$

where: $B(r, s)$ - structuring element in dimensions: $n \times n, 0 \leq r, s \leq n-1$, whereas the erosion of this image can be described as:

$$
E(J(x, y), B(r, s))=\max _{r, s \in B(x, y)}\{J(x+r, y+s)-B(r, s)\}
$$


Taking into account the equations $(1,2)$, the morphological opening of the intensity image $J(x, y)$ with the structuring element $B(r, s)$ is defined with the following formula:

$$
O(J(x, y), B(r, s))=D(E(J(x, y), B(r, s)), B(r, s)) .
$$

\subsection{Global thresholding methods}

To binarize the thermal image of investigated surface, in paper two thresholding methods are used. The first method is based on the image histogram analysis and the second one is the Otsu method. The binarization is an operation, which converts an intensity image into the binary image. The essential is the appropriate selection of the threshold value. In the first method used in this work, the threshold value is selected on the basis of the image histogram. In this paper the Otsu method is used as well. Otsu method is a technique formulated on the basis of the discriminatory analysis. Using this method the image is divided into the two classes (the objects and the background). Otsu method utilizes the probability analysis of the particular classes for obtaining the suitable threshold value.

The binarization of the image $J(x, y)$ is defined as follows [6]:

$$
J_{B}(x, y)=\left\{\begin{array}{ll}
1 & J(x, y)>v_{t h} \\
0 & J(x, y) \leq v_{t h}
\end{array},\right.
$$

where: $J_{B}(x, y)$ - binary image, $v_{t h}-$ threshold value.

The Otsu method was defined on the basis of the statistical image analysis. The calculated threshold value $v_{t h}$ divides the pixels into the two subsets: $c_{0}$ attributed to the background (non-defect areas) and $c_{1}$ attributed to object (defects). The fundamental concept used in the Otsu method is the normalized histogram. It can be treated as the probability distribution [7].

The optimal selection of the threshold value $v_{t h}{ }^{*}$, consists in finding out the maximum of the function $\eta\left(v_{t h}\right)$ :

$$
v_{t h}^{*}=\arg \max _{0<v_{t h}<L-1} \eta\left(v_{t h}\right)
$$

where: $L-1$ - maximal intensity level, existing in the analyzed image. The maximized function $\eta\left(v_{t h}\right)$ is defined as:

$$
\eta\left(v_{t h}\right)=\frac{\sigma_{B}^{2}}{\sigma_{T}^{2}},
$$

where: $\sigma_{B}^{2}-$ between-groups variance, $\sigma_{T}^{2}-$ total variance.

If in the histogram of the analyzed thermal image the two well-defined peaks (the background and the defect) can be distinct, the choice of the threshold value is quite simple. In such a case the value between the peaks is accepted most frequently. Unfortunately, for defect with the low thermal contrast (for example deeply localized) or the thermal images which contain the noise, in the histogram only one peak exists. In this work, in order to calculation of the optimal threshold value, the modified method for the histogram analysis was used. It is sometimes called "the triangle method" [7].

In fig. 1 it is presented the exemplary one-peak histogram of the thermal image.

Fig. 1. An illustration of the triangle method for the determination of the threshold value in case of one-peak histogram [7] 
In order to obtain the threshold value, the straight line, linking the maximum and minimum histogram value is constructed. Assuming, that the histogram minimum $h_{\text {min }}$ lies in the point with coordinates $\left(i_{h \text { min }}, n_{h \text { min }}\right)$, and the histogram maximum $h_{\max }$, in the point with coordinates $\left(i_{h_{-} \max }, n_{h_{\_} \max }\right)$, the desired line can be described, using the following general equation:

$$
A i+B n_{i}+C=0
$$

where: $A, B, C$ - constants, $i$ - intensity level, $n_{i}$ - the number of the pixels with the $i$-th intensity level. The distance of the line (7) from the point with $\left(i_{h}, n_{h}\right)$ coordinates can be expressed as:

$$
d\left(A, B, C, i_{h}, n_{h}\right)=\frac{\left|A i_{h}+B n_{h}+C\right|}{\sqrt{A^{2}+B^{2}}} .
$$

Finally, the optimal threshold value is set as equals to the intensity for which the distance (8) between the line (7) and the histogram over the intensity range $i \in\left(i_{h_{-} \min }, i_{h_{-} \max }\right)$ is the largest. This condition can be defined by the formula:

$$
v_{t h}^{*}=\arg \max _{i} d \text { for } i \in\left(i_{h_{-} \min }, i_{h_{-} \max }\right) .
$$

\section{Experimental investigations}

\subsection{Measuring position}

The experimental research were carried out using the experimental setup presented in fig. 2. It consists of the LW infrared camera FLIR ThermaCAM PM595 (1) with suitable digital interface (2), and the PC (3) with the frame grabber card and the software for the real-time acquisition of the thermal image sequences [4]. As a thermal excitation, two heating lamps (4) were used, each of the $250 \mathrm{~W}$ power. The sample under investigation (5) was made of Plexiglas. The surface of the sample was painted with the black paint with the high emissivity $(\varepsilon \approx 0,98)$. At the bottom side, the four holes, each of ten mm in diameter, were drilled out. The simulated defects were localized in the following depths: $z_{1}=0.5 \mathrm{~mm}, z_{2}=1.5 \mathrm{~mm}, z_{3}=2.5$ $\mathrm{mm} \mathrm{i} z_{4}=3.5 \mathrm{~mm}$ from the top of the sample. Dimensions of the sample and the localizations of defects are presented in fig. 3 .

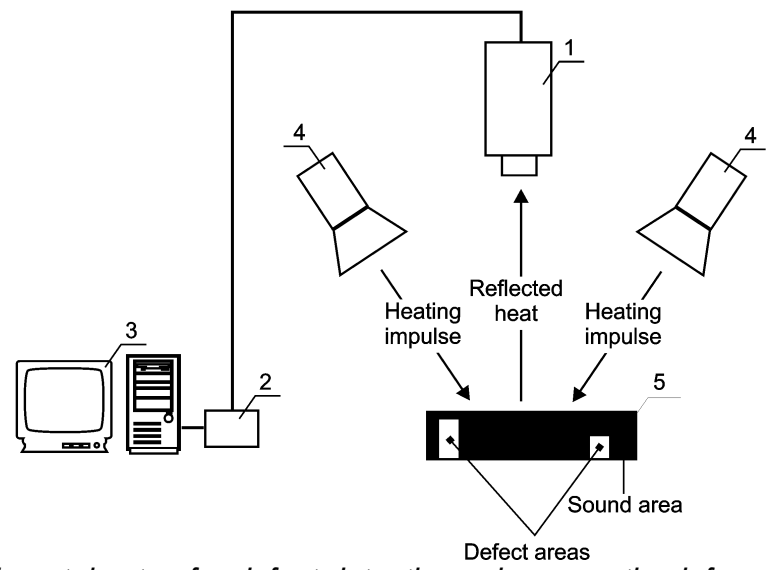

Fig. 2. The experimental setup for defect detection using an active infrared thermography [4]

\subsection{Results of the experimental investigations}

In the research, the stepped heating method was used [1]. Investigated sample was subjected to the heating pulse for $120 \mathrm{~s}$. Next, the sample was cooling down. Thermal image sequence was recorded for $120 \mathrm{~s}$ of heating phase and $120 \mathrm{~s}$ of cooling. The frame rate of recording was assumed as $5 \mathrm{~Hz}$ and the total number of the recorded thermal images was equal to 1200 . The exemplary results of experiments are presented below. The thermal image recorded for time $t=120 \mathrm{~s}$ 
(end of the heating phase) is presented in Fig. 4. Similarly, the thermal image for $t=180 \mathrm{~s}$ (a half time of observation of the cooling phase) is presented in Fig. 5.

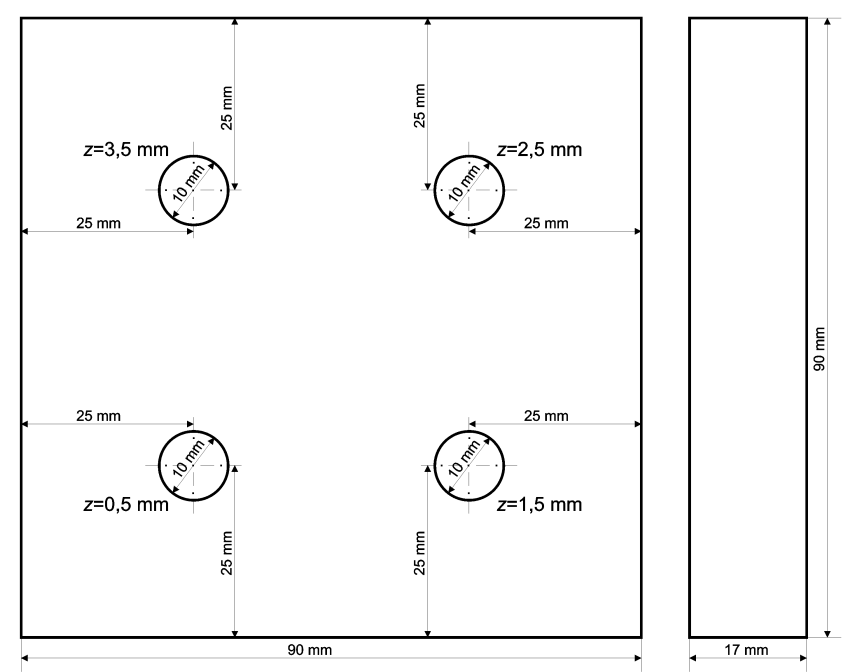

Fig. 3. The locations and dimensions of defects

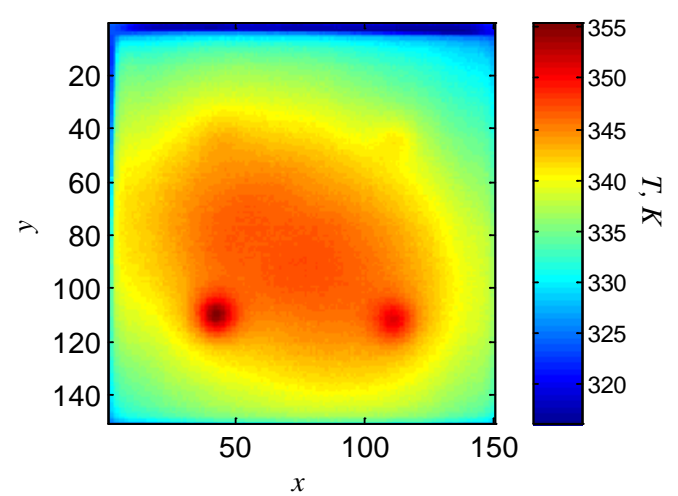

Fig. 4. The thermal image of investigated sample recorded for $t=120 \mathrm{~s}$

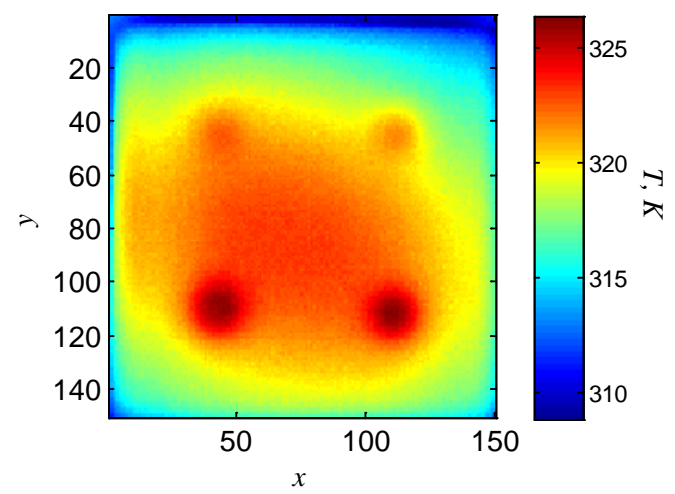

Fig. 5. The thermal image of investigated sample recorded for $t=180 \mathrm{~s}$

4. Simulation research

4.1 Image processing procedure 
In the simulation research, the following stages of the image processing procedure were applied:

1. Storing the thermal image sequence into the three-dimensional temperature matrix $S(x, y, z)$, where: $x, y-$ thermal image size in $x$ and $y$ direction, $z$ - number of the image in the sequence.

2. Choosing of the suitable thermal image to processing.

3. Linear mapping of thermal image from range of $\left(T_{\min }, T_{\max }\right)$, to range of $(0,255)$ - conversion into the intensity image.

4. Estimation of the non-uniform background with the morphological opening operation for different size of the structuring element.

5. Creating the differential image $J_{b-}(x, y)$ by subtraction the estimated background from the original image.

6. Thresholding of the $J_{b-}(x, y)$ image, using two methods mentioned above.

7. Comparison of the results of the background estimation and image thresholding.

The thermal image processing procedure was carried out using the software MORPHGUI. It was created in the MATLAB environment and the Image Processing Toolbox.

The basic data format, used in the Image Processing Toolbox is an intensity image format. The intensity image is implemented as a matrix containing the integer numbers, ranging from 0 to 255 (called the intensity levels) [8]. In the simulations the thermal images were converted into the intensity images, using the following formula:

$$
J(x, y)=\frac{255}{T_{S \max }-T_{S \min }}\left[T(x, y)-T_{S \min }\right]
$$

where: $J(x, y)$ - intensity level of the pixel with $(x, y)$ coordinates, corresponding to the thermal image pixel, indicating the temperature $T(x, y), T_{\mathrm{Smax}}, T_{\mathrm{Smin}}$ - maximal and minimal values of temperature over all the thermal images in the investigated sequence.

\subsection{Results of simulations}

Below, the exemplary results of the thermal image processing were presented. They were obtained for the thermal image recorded in the experimental investigations, presented in fig. 5. The image was processed using the software and the image processing procedure presented above. The results of the background estimation using the opening with the diskshaped of 15 pixels in diameter structuring element is presented in Fig. 6 . In addition, the $J_{b}(x, y)$ image (Fig. 7$)$ and the binary image (Fig. 8), thresholded with the Otsu method are presented as well.

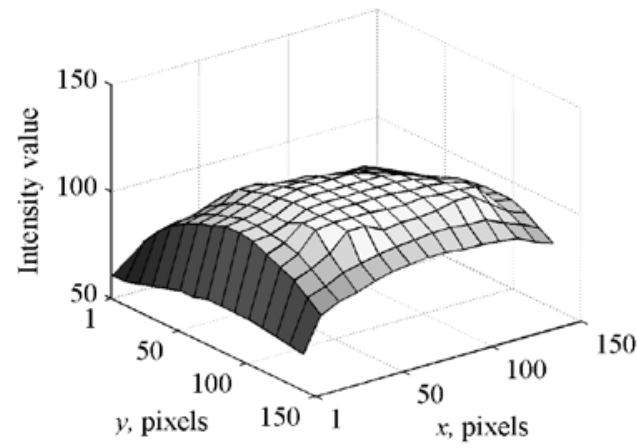

Fig. 6. The estimated background (morphological opening with disk-shaped structuring element, 15 pixels in diameter) 


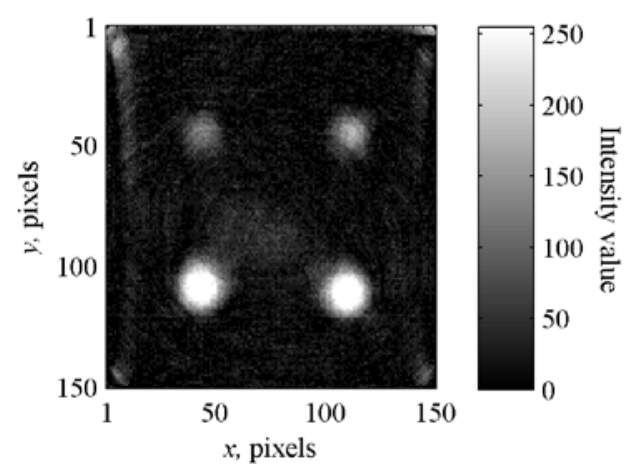

Fig. 7. The image $J_{b-}(x, y)$ (originated after the background subtraction)

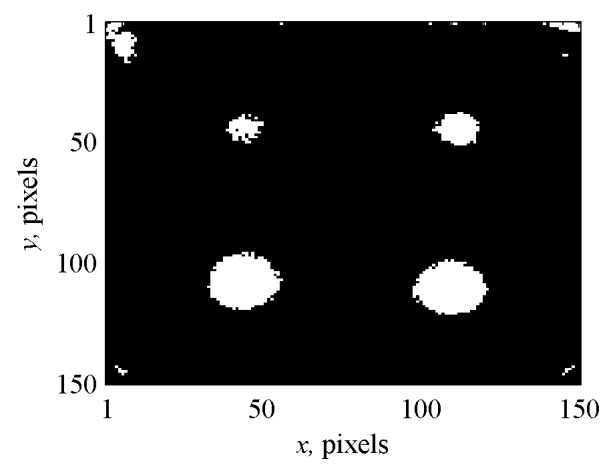

Fig. 7. The binary image (created using Otsu method)

\section{Conclusions}

In this paper the examples of the application of the morphological operations to the estimation of the non-uniform thermal background were presented. The estimation was performed for the testing sample containing the subsurface defects with different depths. In the investigations, the thermal image processing procedure was proposed. This procedure uses the morphological opening operation in order to detect the defects. In the first stage, the experimental investigations were carried out. Analyzing the results of the experiments, the following conclusions can be drawn:

- Comparing the recorded thermal images, it is necessary to state, that the defects which are closer to the top surface of the investigated sample are better visualized in the early phase of the heating, contrary to the deeper defects. In order to observe the deeper defects, the heating time should be increased.

- Te deepest defects (i.e. $z_{3}=2.5$ and $z_{4}=3.5 \mathrm{~mm}$ in depth) become visible only when the sample is cooling down (i.e. in the cooling phase).

- $\quad$ Both in the heating and the cooling phase, the strongly non-uniform thermal background can be observed.

On the basis of the simulation research, the following conclusions were drawn:

- The results of the thermal image processing using the described procedure strongly depend on the shape and the size of the structuring element.

- $\quad$ For thermal images recorded in this work, the better results of defect detection were obtained with the disk-shaped structuring element of 15 pixels in diameter. It approximately corresponds to the real defect diameter, calculated using the field of view of used infrared camera.

- Taking into account the results obtained in this work, the histogram analysis method for the thermal images thresholding can be recommended. This method gives the similar results as the Otsu method, but it is much simpler in the implementation and more efficient. 


\section{REFERENCES}

[1] X.P. Maldague, "Theory and practice of infrared technology for nondestructive testing", John Wiley \& Sons Interscience, New York (2001).

[2] A. Gleiter, C. Spiessberger, G. Busse, "Phase angle thermography for depth resolved characterization", Proc. 9th International Conference on Quantitative Infrared Thermography QiRT, July 2-5, Cracow - Poland, pp. $435-441$ (2008).

[3] C. Zöcke, A. Langmeier, R. Sţ̧ßbel, W. Arnold, "Reconstruction of the defect shape from lock -in thermography phase images", QIRT Journal, Vol. 6, No. 1/2009, pp. 63-78 (2009).

[4] S. Dudzik, "A simple method for defect area detection using active thermography", Opto-Electronics Review, Vol. 17, Issue 4, pp. 338-344, DOI: 10.2478/s11772-009-0016-9v (2009).

[5] W. Minkina, S. Dudzik, "Infrared Thermography - Errors and Uncertainties", John Wiley \& Sons, Chichester, 2009 r., ISBN-10: 0470747188, ISBN-13: 978-0470747186

[6] J.C. Russ, "Image Processing Handbook", CRC Press LLC (2002).

[7] J. Serre, „Image analysis and mathematical morphology”, Academic Press, New York (1982).

[8] The Image Processing Toolbox Users' Guide for MATLAB R2009b, The MathWorks, Inc. (2009). 